\title{
Structure-function relationships in mitochondrial transcriptional condensates
}

\author{
Marina Feric ${ }^{1,2}$, Azadeh Sarfallah ${ }^{3} \dagger$, Furqan Dar ${ }^{4}$, Dmitry Temiakov ${ }^{3}$, Rohit V. Pappu ${ }^{5}$, Tom \\ Misteli $^{1}$
}

\begin{abstract}
Affiliations
${ }^{1}$ National Cancer Institute, National Institutes of Health, Bethesda, MD, 20892, USA

${ }^{2}$ National Institute of General Medical Sciences (NIGMS), National Institutes of Health, Bethesda, MD, 20892, USA

${ }^{3}$ Department of Biochemistry and Molecular Biology, Thomas Jefferson University, 1020 Locust Street, Philadelphia, PA 19107, USA

${ }^{4}$ Department of Physics, Washington University in St. Louis, St. Louis, MO 63130, USA

${ }^{5}$ Department of Biomedical Engineering, Center for Science \& Engineering of Living Systems (CSELS), Washington University in St. Louis, St. Louis, MO 63130, USA

*Correspondence to: marina.feric@,nih.gov; mistelit@mail.nih.gov

$\dagger$ Current address: Department of Biochemistry and Biophysics, Perelman School of Medicine, University of Pennsylvania, Philadelphia, PA 19104
\end{abstract}

\section{Key words}

biomolecular condensates, phase separation, transcription, mitochondrial genome, mitochondrial nucleoids, mitochondria, vesicles, TFAM, POLRMT 


\begin{abstract}
Phase separation organizes many membraneless structures in cells. The functional consequences of concentrating cellular machinery into biomolecular condensates, however, is largely unclear. Here, we use in vitro reconstitutions, in vivo studies, and computational modelling to uncover structure-function relationships of mitochondrial (mt-) transcriptional condensates. In vitro, we find that the core mt-transcription machinery - consisting of POLRMT, TFAM, TFB2M, and DNA - forms viscoelastic, multi-phasic condensates. Strikingly, the rates of condensate-mediated transcription are considerably lower than equivalent reactions in bulk solution. Dampened rates are associated with reduced diffusivities of components that become kinetically arrested in non-equilibrium, vesicular condensates. Perturbation of mt-components in vivo and computational simulations recapitulate the transcription-dependent reorganizations observed in vitro. Our findings demonstrate close, bidirectional interdependence between structure and function of transcriptional condensates.
\end{abstract}

One-sentence summary

Production of RNA alters phase behavior of mitochondrial transcriptional condensates. 


\section{Introduction}

Proteins and nucleic acids form diverse biomolecular condensates which arise via phase separation (1-4). Condensates emerge by de-mixing of protein, RNA and DNA components from their cellular surroundings to form distinct, non-membrane bound cellular structures. The formation of condensates is typically mediated by multivalent homotypic and heterotypic interactions amongst proteins and nucleic acids (1). Prominent biomolecular condensates include P-granules and stress granules in the cytoplasm $(5,6)$ as well as the nucleolus and RNA splicing factor speckles in the nucleus $(7,8)$. In mitochondria, the genome-containing nucleoid and RNA processing granules are condensates formed by phase separation $(9,10)$.

Condensates are enriched in functional components, such as transcription factors or RNA processing factors, and their concentration in phase separated structures may contribute to enhanced reaction rates of relevant processes within condensates (11). However, the connection between condensate structure and function remains unclear (2). A major hurdle in elucidating structure-function relationships of condensates has been the difficulty to reconstitute functionally active in vitro condensates that contain all relevant components. Conversely, in vivo perturbations, such as mutagenesis or pharmacological disruption, make it challenging to directly isolate the effects of phase behavior from the functional properties of the affected cellular components.

Condensates are thought to contribute to many cellular functions including in transcription and genome organization (12-14). Major architectural chromatin proteins such as the linker histone $\mathrm{H} 1$ (15) and the heterochromatin protein HP $1 \alpha$ form condensates in vitro and in vivo (16, 17). Phase separation, in different manifestations, has been suggested to contribute to higher-order organization of genomes into domains and compartments (18-20). In particular, various components of the transcription machinery spontaneously concentrate into condensed phases in the mammalian nucleus, including at sites of super-enhancers (21). This behavior has been attributed to the presence of intrinsically disordered regions (IDRs) in many transcription factors and chromatin proteins (22). These are thought to mediate weak, multivalent protein-protein interactions that give rise to dynamic, non-stoichiometric condensed assemblies (23). Functionally, the condensation of transcription components is associated with bursts of transcription of RNA (24) that are consequently followed by dissolution of the condensate in an effective feedback loop (25).

The mitochondrial (mt) genome (mtDNA) and its gene expression machinery are also organized via phase separation (9). Human mitochondria contain a separate $16 \mathrm{~kb}$, circular genome (26) that assembles into mitochondrial nucleoids, which are membraneless, nucleoprotein complexes of $\sim 100 \mathrm{~nm}$ in diameter containing mtDNA and its associated proteins $(27,28)$. The major mt-genome architectural protein TFAM phase separates in vitro and in vivo, and combined with mtDNA, forms condensates that recapitulate the dynamic behavior of mt-nucleoids in cells (9). In addition to mitochondrial transcription, processing of newly synthesized mtRNA occurs in adjacent phase separated RNA granules associated with the mitochondrial membrane (10).

The relative simplicity, involving only a small number of minimally required components, makes mitochondrial transcription a unique model system to probe structure-function relationships in a biologically relevant condensate. Mitochondrial transcription can be reconstituted under soluble 
conditions with only four components: mtDNA, the single-subunit mitochondrial RNA polymerase POLRMT, and two transcription factors TFAM and TFB2M $(29,30)$. Here, we have reconstituted mitochondrial transcription under condensate-forming conditions as it occurs in vivo, and we directly probe functional consequences of the condensate morphologies and their internal physicochemical environments. We demonstrate that the mitochondrial transcription machinery forms multi-phasic, transcriptionally active, non-equilibrium condensates with slow diffusivities, resulting in dampened transcriptional kinetics compared to equivalent reactions in bulk solutions. Our results demonstrate a close interplay between the physical behavior and functional activity of an archetypal biomolecular condensate.

\section{Results}

\section{Mitochondrial transcription machinery phase separates into heterogeneous droplets}

Mitochondrial transcription can be reconstituted with three proteins and a DNA template under dilute conditions (30). We first sought to establish the phase behavior of the minimal components required for mitochondrial transcription. Structural studies (31) and bioinformatics analysis reveal that the minimally required human mitochondrial transcription components TFAM, TFB2M, and POLRMT all contain a combination of ordered functional domains and IDRs (Fig. 1A). Computational predictions for disordered proteins suggest that unbound TFAM is the most disordered of the three proteins with a flexible linker that bridges two DNA binding domains (High Mobility Groups A, B) and a disordered C-terminus. This is consistent with the flexible nature and multiple conformations that have been reported for TFAM molecules in solution (32). TFB2M and POLRMT are significantly more structured $(29,33)$, but both contain disordered N-termini that that have the potential to contribute to phase separation.

We find that the mitochondrial proteins TFAM, TFB2M, and POLRMT and mtDNA individually de-mix from solution and form dense condensates under appropriate conditions $(10 \mu \mathrm{M}$ TFAM/TFB2M in 10\% PEG; $1.5 \mu \mathrm{M}$ POLRMT in 5\% PEG; $500 \mathrm{nM}$ DNA in 10\% PEG) (Fig. 1B; Fig. S1). Condensates formed by TFAM, TFB2M, or mtDNA each resemble highly spherical droplets with an aspect ratio of $\sim 1$, consistent with phase separation (Fig. 1B; Fig. S1). In contrast, POLRMT assembles into highly irregular structures (Fig. 1B; Fig. S1). These are likely to be dynamically arrested phases in which strong intermolecular interactions arrest phase separation $(34,35)$. Our results show that all minimal components of the mitochondrial transcription machinery can individually self-associate and form higher-order assemblies under the influence of homotypic interactions.

Next, we dissected the co-phase behavior of multiple components by characterizing the structures formed by binary and ternary mixtures of components of the minimal mitochondrial transcription machinery (Fig. 1C). Using the correlation coefficient as a metric of their colocalization, we find that TFAM and DNA form multi-phase condensates, containing micron-sized sub-domains that are either TFAM-rich or DNA-rich (Fig. 1C). TFB2M and POLRMT show lower degrees of colocalization (Fig. 1C). When the proteins were mixed in pairs without DNA, TFAM and TFB2M colocalize with one another although they do not colocalize as well with POLRMT (Fig. 1C).

For ternary combinations of pairs of proteins with DNA, the droplet organization remained heterogeneous for all combinations, with micron-sized domains forming for TFAM-TFB2M-DNA 
and TFB2M-POLRMT-DNA (Fig. 1C). However, for the ternary mixture of all proteins without DNA, the condensates became more well-mixed, implying that the heterotypic interactions of proteins with DNA contribute to the emergence of coexisting phases of the compound droplets (Fig. 1C). These results demonstrate differential phase behavior of the various components of the mitochondrial transcription machinery.

To reconstitute transcriptionally competent condensates, all four components (DNA, POLRMT, TFAM and TFB2M) were combined in equimolar ratios ( $\sim \mu \mathrm{M}$ TFAM, TFB2M, and POLRMT with $500 \mathrm{nM}$ DNA in 5\% PEG) (Fig. 1D). In this quaternary mixture, all components de-mixed into single droplets, which often contacted the neighboring droplets to form dynamically arrested higher-order structures (Fig. 1D). Moreover, the localization of individual components within the multi-phasic structure remained heterogeneous: POLRMT showed the lowest level of colocalization with all other components, and TFB2M tended to accumulate more peripherally (Fig. 1D). Importantly, the localization correlation coefficients for specific pairs tended to be higher in the compound droplet than those found in the binary or ternary droplets (Fig. 1C-D). This behavior is consistent with the dual-nature of TFAM, which has affinity for DNA via its strong N-terminal DNA-binding domain (HMGA), but also for other proteins, such as POLRMT, via its disordered C-terminus (9). Contrary to what would be expected for purely viscous liquids, quaternary condensates did not relax into spherical structures. Instead, they persisted as an amalgam of smaller droplets, beaded together (Fig. 1D) (36). The inability to rearrange into a single spherical droplet suggests that the condensates are dynamically arrested phases that arise due heterogeneities in the strengths of intermolecular homotypic vs. heterotypic interactions (37). Taken together these results show that the minimal mitochondrial transcription machinery can collectively form multi-phasic, dynamically arrested, condensates in vitro.

\section{Condensates support transcription and dampen transcription rates}

Next, we tested whether the in vitro condensates formed by the four macromolecular components could support transcription under condensate-forming conditions. To do so, we added a full set of nucleotides (NTPs) to the quaternary mixture of mtDNA, POLRMT, TFAM, TFB2M and incubating the reaction at $\sim 35^{\circ} \mathrm{C}$ (see Methods). After 30 minutes of incubation, transcriptional activity was measured by detection of a $\sim 300$ nt RNA product using a PCR-amplified template containing the LSP promoter and radioactively labeled nucleotides as previously described (38) (Fig. 2A). As a control, robust transcription was detected and increased roughly linearly under soluble conditions (-PEG) over a $\sim 7$-fold range of initial concentrations $(0.6 \mathrm{mM}$ TFAM, $0.6 \mathrm{mM}$ TFB2M, $0.6 \mathrm{mM}$ POLRMT, and $50 \mathrm{nM}$ DNA) before dropping off at higher concentrations of components (Fig. 2A, B). Similarly, quaternary condensates comprising all transcriptional components were transcriptionally active (Fig. 2A). However, in contrast to the corresponding bulk conditions, transcriptional output in condensates was reduced between 1.3-fold (10X condition) and 20-fold (1X condition) (Fig. 2B, C). Dampening of transcriptional output in condensates occurred first at $1 \mathrm{X}$ concentrations of components and approached unity with increasing reactant concentration (Fig. 2A-C). These results demonstrate transcriptional activity in reconstituted mitochondrial condensates and that transcription is dampened in condensates compared to in solution.

To confirm the formation of condensates under transcription conditions and to relate condensate structure to function, we compared the condensate morphologies under conditions of various 
component concentrations (Fig. 2D, E; Fig. S2). As expected, in the absence of crowder, the most dilute case $(1 \mathrm{X},-\mathrm{PEG})$ was fully soluble, and no condensates nor protein precipitates were observed (Fig. S2B-E). In contrast, under transcriptionally competent, condensate-forming conditions (1X-10X, +PEG), RNA and all transcriptional components localized to the periphery of condensates, resembling vesicle-like morphologies as visualized after 60 minutes of reaction (Fig. 2D; Fig. S2B-E, ref. (39)). In these vesicles, DNA tended to associate to the outermost regions, whereas RNA and proteins co-localized in the interior shell (Fig. S2). The peripheral localization of the transcription machinery was a consequence of active transcription in condensates, since it did not occur in identical, but transcription incompetent, condensates generated in the presence of only UTP nucleotides (Fig. 2E-G; see below). Our observation that newly synthesized RNA, and the associated transcription machinery, accumulate at the periphery of mitochondrial condensates upon transcriptional activity in vitro indicates that the process of transcription leads to internal re-organization of condensate components.

\section{Newly synthesized RNA shapes condensate structure}

To monitor the transcription-dependent re-organization of condensate components over time and to test whether RNA is exclusively produced at the edge of the condensates or whether RNA accumulates over time at the condensate periphery, we performed time-course experiments (Fig. 3). RNA can be detected as early as 5 minutes in the condensate interior (Fig. 3A, B), but the condensates at early time points of 5 and 10 minutes do not resemble vesicles but retain the transcription machinery components throughout the interior (Fig. 3A, B). Moreover, RNA at these early time points is predominantly located internally, with only slight accumulation near the periphery. In contrast, at 20, 40, and 60 minutes, we detect a significant change in the organization of the condensate, where pronounced vacuoles start to appear within the condensates, leading to formation of a peripheral shell containing the RNA and transcription components and to increased vacuole size with time (Fig. 3A, B). All the components become peripherally located with increasing reaction time concomitant with production of RNA (Fig. 3A, B). The observed reorganization of transcription components during the reaction suggests that the process of generating RNA modulates structural changes of condensates.

To further test the role of internally generated RNA in determining condensate structure and to address whether the vesicles generated during the in vitro reaction are equilibrium structures or metastable arrested phases that arise due to increased levels of RNA, we added exogenous RNA comparable in sequence and length (ex-RNA) to the mitochondrial components at various time points in the presence of only UTPs to prevent transcription in the condensate. Addition of exRNA at the beginning of the reaction resulted in droplets with a layered structure, where ex-RNA, TFAM and POLRMT were in the interior, surrounded by a layer of DNA (Fig. 3C). In contrast, addition of ex-RNA after the condensates had assembled $(\mathrm{t}=5,30 \mathrm{~min})$, resulted in droplets that had the reverse layered organization, where DNA, TFAM, and POLRMT were in the interior, surrounded by a peripheral layer of ex-RNA (Fig. 3C). We found that in all these cases, TFAM and POLRMT tended to partition with ex-RNA, suggesting protein-RNA interactions are energetically favorable. However, in all these cases, there was little colocalization of DNA with RNA, implying that DNA and RNA repel each other, making these interactions energetically unfavorable. The fact that we obtain different organizations of the components depending on whether RNA is added to or is generated within the condensate confirms a role for active 
transcription in shaping condensate structure and suggests that active mt-transcriptional condensates are non-equilibrium, dynamically arrested structures.

Given that the condensates take on vesicle-like morphologies that appear dynamically arrested and transcription rates are suppressed when reactions occur within condensates, we sought to directly characterize the molecular dynamics within transcriptional condensates. Fluorescence recovery after photobleaching (FRAP) analysis of RNA detected by the incorporated fluorescently labeled nucleotides after a $\sim 30$ min reaction demonstrated minimal recovery over the course of $\sim 15$ minutes (Fig. 3D), indicating very slow diffusivity in the interior of the condensate. As a control, free nucleotides in a transcription-incompetent condensate rapidly exchanged resulting in only limited bleach depth (Fig. S3A). Taken together, the slow internal dynamics directly support that the reconstituted condensates become dynamically arrested upon transcription.

\section{Perturbation of mitochondrial structure in vivo recapitulates aspects of transcription- dependent reorganization}

We next extended our in vitro studies on mito-transcriptional condensates to test how their observed phase behavior relates to in vivo conditions. Normally, mt-nucleoids assemble into membraneless, nucleoprotein complexes of typically $\sim 100 \mathrm{~nm}$ in diameter that are distributed throughout the mitochondrial network (Fig. 4A) (27). The mt-nucleoids are sites of transcription, and the newly transcribed polycistronic mtRNA localizes to adjacent phase-separated mitochondrial RNA granules for further processing $(10,40)$. To probe structure-function relationships of mitochondrial condensates in vivo, we depleted key mitochondrial transcription components and assessed their effects on mt-nucleoid fine-structure.

Partial knockdown of TFAM by RNAi (Fig. S4B) led to a significant reorganization of mtnucleoids as previously observed in TFAM heterozygous knockout mice (41). The number of mtnucleoids per cell, based on staining for TFAM and DNA, was reduced significantly. In line with prior observations of induction of a stress response involving increased interferon stimulated gene expression and enhanced type I interferon responses upon loss of TFAM (41), a few, dramatically enlarged mt-nucleoids were observed in TFAM depleted HeLa cells (Fig. 4B). Interestingly, these remaining stress-induced mt-nucleoids resembled the heterogeneous condensates observed in vitro (9), where TFAM and mtDNA formed $>1$ micron-sized, multiphase droplets. Indeed, these enlarged nucleoids allowed us to spatially resolve the organization of the mitochondrial (ribo)nucleoprotein complexes (Fig. 4B). As observed in vitro under condensate forming conditions we found that $12 \mathrm{~S}$ RNA localized peripherally and de-mixed from TFAM and mtDNA (Fig. 4B), supporting the conclusion that mt-nucleoids and mtRNA exist as spatially distinct phases in live mitochondria.

The mt-nucleoid-associated protein MTERF2 (mitochondrial transcription termination factor 2), is an abundant mt-nucleoid protein, present at $\sim 100: 1$ copies relative to mtDNA (42). Partial knockdown of MTERF2 also led to a stress response (Fig. S4B), resulting in a population of swollen mitochondria that allowed us to spatially resolve these altered (ribo)nucleoprotein complexes. The swollen mitochondria showed a ring-like accumulation of RNA (Fig. 4C), reminiscent of the shells we observe under reacting, crowded conditions in vitro (Fig. 2). TFAM accumulated in these mitochondria and appeared to wet the inner surface of 12S RNA. However, 
mtDNA remained organized as $\sim 100 \mathrm{~nm}$ puncta surrounded by TFAM and that frequently colocalized with bright puncta of 12S RNA (Fig. 4C).

Finally, using a mitochondrially targeted endonuclease (43), depletion of mtDNA led to complete dissolution of mt-nucleoids - including loss of TFAM signal (Fig. S4). The dissolution of DNAand RNA-rich condensates in the inner mitochondrial matrix upon mtDNA depletion suggests that the mt-DNA nucleates mitochondrial transcriptional condensates in live cells.

Taken together, our results support the notion that the (ribo)nucleoprotein complexes in the mitochondrial matrix represent coexisting phases, whose organization is modulated by the physicochemical nature of the constituents as well as functional activity of the mt-nucleoid, specifically RNA production during active transcription.

\section{Computational simulation of transcription-mediated reorganization}

We next thought to explore the mechanisms by which newly synthesized RNA re-organizes the non-equilibrium condensates in vitro and how these effects relate to the steady-state conditions found in vivo (Fig. 3C). For this, we turned to computational modeling, using the simulation engine LaSSI (44). We performed Monte-Carlo (MC) simulations of coarse-grained (CG) models of binary and higher-order mixtures to probe the effects of RNA production on equilibrium condensates. To preserve the overall length-scales and interaction hierarchies of the macromolecules involved in transcriptionally active condensates in the simulations, DNA molecules were modeled as chains of 20 beads, RNA as chains of 10 beads, TFAM and TFB2M as chains of 4 beads, POLRMT as chains of 3 beads, and crowders as chains of 4 beads (Fig. 5A).

We first parameterized the contact energies between pairs of molecules by reproducing experimentally observed morphologies of single and binary mixtures (Fig. 5C, compare to Fig. 1B, C) (9). The experimentally measured colocalization and condensate homogeneity were used as proxies for stronger heterotypic interactions, while spatial inhomogeneities within the condensates were used as proxies for stronger homotypic interactions. TFAM has an additional anisotropic interaction to account for its weak dimerization upon DNA binding (Fig. 5B) (45). Simulations of the binary combinations of transcriptional condensate components generated morphologies (Fig. 5C; Fig. S5A) with spatial organizations, quantified in terms of radial density profiles, that recapitulate the experimental results for binary mixtures (Fig. 1C). For example, TFAM-DNA and POLRMT-DNA formed multi-phase droplets, while TFB2M and DNA behaved as distinct coexisting phases, and pairs of proteins tended to form well-mixed droplets, as observed in vitro (Fig. 1C, Fig 5C).

Extending this modeling approach to the quaternary condensates, we find heterogenous droplets consistent with our experimental results (Fig. 5E, $\mathrm{t}=0$; compare to Fig. 1D). Simulations produced the formation of layered droplets with DNA being localized almost exclusively at the periphery (Fig. 5D; Fig. S5B). The three proteins tend to be enriched in the interior of the droplet, enveloped by a shell of DNA. This multi-phase organization is consistent with the heterogeneity and colocalization of components in the four-component dynamically arrested droplets observed in vitro (Fig. 1D). 
Based on our experimental observation of re-organization of transcriptional components upon synthesis of nascent RNA in the condensate, we sought to explore how transcription affects condensates. At the initial time point $t_{0}$, all reactants are fully mixed and no RNA is present, while at some later time, $t_{i}>0$, the system contains RNA, $[R]_{i>0}$; conversely, if the system has an amount of RNA, $[R]_{i}$, then the system is at $t_{i}$. Therefore, titrating the amount of RNA in the simulations allowed us to ask what the equilibrium structures of condensates would be when transcription occurs (Fig. 5). We find that owing to the favorable interactions between the RNA and the proteins, newly synthesized RNA is readily incorporated into the existing droplet (Fig. 5E). RNA distributes itself into the interior of the droplets (Fig. 5D; Fig. S5B), and with increasing levels of RNA, TFB2M is peripheralized from the interior, which further pushes DNA to be localized almost exclusively to the periphery (Fig. 5D). Therefore, in accord with experimental observations, the simulations show that transcription directly remodels the organization of the condensate components. Strikingly, the computationally predicted equilibrium structures are exact facsimiles of structures we obtain upon mixing ex-RNA with components early on, $\mathrm{t}=0$ (Fig. 3C).

While we find that RNA accumulates at the periphery of condensates in vitro, in intact cells newly synthesized RNA is rapidly bound by RNA-binding proteins and processed in mitochondrial RNA granules, which are phase separated structures and are often located adjacent to mt-nucleoids (10, 40). To test whether the action of RNA-binding proteins can account for the distinct RNA distribution in DNA-rich condensates in vitro and in vivo, we incorporated an additional favorable interaction between the crowder and the RNA in our simulations to mimic the effects of association of RNA-binding proteins to the newly synthesized RNA under steady-state conditions in vivo (Fig. 5B). Under these assumptions, the reorganization of transcriptional components mirrors their distribution in vivo, where RNA no longer accumulates within the DNA-rich droplet, but condenses separately in the bulk (inset Fig. 5D, E; Fig. S5C). Overall, these simulations recapitulate the in vivo behavior of mitochondrial condensates, and they also affirm the inference that vesicular structures observed in vitro are non-equilibrium, dynamically arrested phases.

\section{Discussion}

We report here the reconstitution of transcriptionally active, multi-phasic condensates using the human mitochondrial transcription machinery as a model system. We find that, when compared to bulk reactions in solution, the transcriptional rate is reduced under condensate-forming conditions. We suggest this to be due to the viscoelastic and dynamically arrested nature of the droplets. We also observe that the production of RNA in condensates alters the spatial organization of condensates, thus providing direct evidence for a dynamic interplay between the structure of condensates and the functional activities they harbor.

There are growing accounts of transcription occurring within condensates in vivo. For example, RNA Pol I transcribes rRNA in the multiphase nucleolus (7, 46), RNA Pol II produces mRNA in transcriptional condensates throughout the nucleus $(47,48)$, and POLRMT generates long, polycistronic mtRNA in mitochondrial condensates $(9,10,31)$. While condensate formation is not an absolute requirement for transcription since transcription can be reconstituted in vitro under conditions where condensates do not form (49), there is growing evidence that condensate formation may offer unique advantages for regulation of transcription in vivo. First, the condensed phase creates increased concentrations of reactants, which may regulate mass action effects (11). For example, SUMOylation can be increased $\sim 36$ fold in engineered condensates in vitro (50). In 
addition, increased dwell times of proteins associated with the condensate microenvironment can also be conducive for assembly of reacting complexes (51). In support of this hypothesis, an early FRAP study showed that the kinetic behavior of multiple RNA Pol I components could only be explained by inclusion of a slow kinetic component prior to binding of the polymerase subunits to the promoter, possibly reflecting slowed diffusion in the nucleolar condensate (52).

The markedly slower internal dynamics observed here in mitochondrial condensates may suggest that they operate at elevated Damköhler numbers $(D a \sim k / D)$, which compare reaction rates $(k)$ relative to the diffusive mass transfer rates (diffusion coefficient, $D$ ) (53). Whereas in bulk solution, reactants can diffuse quickly, and the rate of transcription is only limited by the speed of the polymerase ( $D a<<1$, reaction-limited), the significantly slow dynamics associated with the condensate environment, as they exist in mitochondrial condensates in vivo, suggest that condensates experience diffusion-limited kinetics ( $D a>>1$, diffusion-limited) (11). Indeed, we observe that, for mitochondrial transcriptional condensates, RNA production occurs with dampened transcription rates relative to bulk solution (Fig. 2A). Our rough estimates suggest that less than 1 round of transcription occurs under crowded conditions indicating that not all DNA templates were actively transcribed. This behavior is in line with the situation in live cells, where a minority $(<5 \%)$ of the mitochondrial nucleoids are actively transcribed at any given time $(54$, $55)$.

Importantly, we find that the structure of the transcriptional condensate is affected by its activity. Production of a new chemical species - RNA - in the DNA- and protein-rich transcription condensates leads to non-equilibrium changes in condensate organization, reflected by the emergence of vesicle-like morphologies. Intriguingly, similar vesicle morphologies have been observed for simple in vitro RNA-protein systems (39), in vivo liquid spherical shells of the DNAand RNA-binding protein TDP-43 (56), and in vitro liquid spherical shells of DNA and poly-LLysine (57), suggesting these structures are thermodynamically accessible in specific regions of protein-nucleic acid phase diagrams. Importantly, while the equilibrium simulations predict the organization of multiphase condensates at the onset of the transcription reaction, they do not produce vesicle-like structures as transcription progresses. This reinforces our conclusion that the vesicles we observe in vitro are non-equilibrium structures.

The observed vesicle formation due to RNA generation in transcriptionally active condensates in vitro differs from that of the canonical mt-nucleoid organization in mitochondria in vivo $(27,28)$, where mt-nucleoids remain as condensed, $100 \mathrm{~nm}$ droplet-like structures, and mtRNA localizes to separate granules that coat the mitochondrial inner membrane and are de-mixed from the mtnucleoid (10). Our simulations, which are based on experimentally determined interaction parameters, suggest that during transcription, mtRNA is tethered to the mt-nucleoid as it is being transcribed by POLRMT but later moves - be it actively or passively - out of the mt-nucleoid into a more energetically favorable phase in the mitochondria. An attractive model, consistent with our observations, is that in vivo the nascent mtRNA is effectively handed off from the mt-nucleoids condensates, from where it is generated, to the mitochondrial RNA processing granule, which are condensates themselves (10).

Our observations regarding mt-transcription condensates are also relevant to other cellular transcription processes. Transcription of ribosomal DNA (rDNA) by RNA Pol I occurs in fibrillar 
centers (FC) in the nucleolus, which unlike the mt-nucleoid, contains enveloping coexisting phases of RNA and proteins (7). Analogously, both the mt-nucleoid and the FC center represent cores of a DNA-rich phase (mtDNA or rDNA, respectively), and the nascently transcribed RNA moves radially outwards, into adjacent mt-RNA granules in mitochondria (40) or towards the dense fibrillar component (DFC) and granular components (GC) in the nucleolus (46), respectively. Similarly, RNA Pol II can self-assemble with the transcriptional machinery to form transcriptional condensates, particularly at super-enhancers, in the mammalian nucleus (47). Interestingly, for RNA Pol II condensates, rapid, local RNA production has been shown to result in complete dissolution of the condensate, underscoring a feedback mechanism between phase behavior and RNA production as also observed here for mitochondrial transcription (25). Similar condensation is also seen in the bacterial nucleoid, where RNAP clusters with transcription factors at specific sites, particularly rDNA, in the bacterial genome $(58,59)$. The common observation that emerges from these observations is that RNA is not retained in DNA-rich phases, pointing to an intrinsic energetic barrier for their mixing (Fig. S6). As such, the ability for genomes to undergo phase separation and organize transcription in condensates seems to be conserved across evolution (13). 


\section{References}

1. S. F. Banani, H. O. Lee, A. A. Hyman, M. K. Rosen, Biomolecular condensates: organizers of cellular biochemistry. Nature Reviews Molecular Cell Biology 18, 285 (2017).

2. Y. Shin, C. P. Brangwynne, Liquid phase condensation in cell physiology and disease. Science 357, (2017).

3. B. R. Sabari, A. Dall'Agnese, R. A. Young, Biomolecular condensates in the nucleus. Trends in biochemical sciences, (2020).

4. S. Boeynaems et al., Protein phase separation: a new phase in cell biology. Trends in cell biology 28, 420-435 (2018).

5. C. P. Brangwynne et al., Germline P granules are liquid droplets that localize by controlled dissolution/condensation. Science 324, 1729-1732 (2009).

6. D. W. Sanders et al., Competing protein-RNA interaction networks control multiphase intracellular organization. Cell 181, 306-324. e328 (2020).

7. M. Feric et al., Coexisting liquid phases underlie nucleolar subcompartments. Cell 165, 1686-1697 (2016).

8. J. A. Greig et al., Arginine-enriched mixed-charge domains provide cohesion for nuclear speckle condensation. Molecular Cell 77, 1237-1250. e1234 (2020).

9. M. Feric et al., Self-assembly of multi-component mitochondrial nucleoids via phase separation. EMBO J 40, e107165 (2021).

10. T. Rey et al., Mitochondrial RNA granules are fluid condensates positioned by membrane dynamics. Nature Cell Biology, 1-7 (2020).

11. A. S. Lyon, W. B. Peeples, M. K. Rosen, A framework for understanding the functions of biomolecular condensates across scales. Nature Reviews Molecular Cell Biology 22, 215235 (2021).

12. D. Hnisz, K. Shrinivas, R. A. Young, A. K. Chakraborty, P. A. Sharp, A phase separation model for transcriptional control. Cell 169, 13-23 (2017).

13. M. Feric, T. Misteli, Phase Separation in Genome Organization across Evolution. Trends in Cell Biology, (2021).

14. H. Wang, M. Han, L. S. Qi, Engineering 3D genome organization. Nature Reviews Genetics 22, 343-360 (2021).

15. B. A. Gibson et al., Organization of Chromatin by Intrinsic and Regulated Phase Separation. Cell, (2019).

16. A. R. Strom et al., Phase separation drives heterochromatin domain formation. Nature 547, 241 (2017).

17. A. G. Larson et al., Liquid droplet formation by HP1 $\alpha$ suggests a role for phase separation in heterochromatin. Nature 547, 236 (2017).

18. M. Falk et al., Heterochromatin drives compartmentalization of inverted and conventional nuclei. Nature 570, 395-399 (2019).

19. F. Erdel, K. Rippe, Formation of chromatin subcompartments by phase separation. Biophysical journal 114, 2262-2270 (2018).

20. H. Strickfaden et al., Condensed Chromatin Behaves like a Solid on the Mesoscale In Vitro and in Living Cells. Cell 183, 1772-1784.e1713 (2020).

21. B. R. Sabari et al., Coactivator condensation at super-enhancers links phase separation and gene control. Science, eaar3958 (2018). 
22. A. Boija et al., Transcription factors activate genes through the phase-separation capacity of their activation domains. Cell 175, 1842-1855. e1816 (2018).

23. S. Chong et al., Imaging dynamic and selective low-complexity domain interactions that control gene transcription. Science 361, eaar2555 (2018).

24. M.-T. Wei et al., Nucleated transcriptional condensates amplify gene expression. Nature cell biology 22, 1187-1196 (2020).

25. J. E. Henninger et al., RNA-mediated feedback control of transcriptional condensates. Cell 184, 207-225. e224 (2021).

26. X. J. Chen, R. A. Butow, The organization and inheritance of the mitochondrial genome. Nature Reviews Genetics 6, 815 (2005).

27. C. Kukat et al., Super-resolution microscopy reveals that mammalian mitochondrial nucleoids have a uniform size and frequently contain a single copy of mtDNA. Proceedings of the National Academy of Sciences 108, 13534-13539 (2011).

28. N. Garrido et al., Composition and dynamics of human mitochondrial nucleoids. Molecular biology of the cell 14, 1583-1596 (2003).

29. H. S. Hillen, Y. I. Morozov, A. Sarfallah, D. Temiakov, P. Cramer, Structural Basis of Mitochondrial Transcription Initiation. Cell 171, 1072-1081.e1010 (2017).

30. D. Litonin et al., Human mitochondrial transcription revisited: only TFAM and TFB2M are required for transcription of the mitochondrial genes in vitro. Journal of Biological Chemistry 285, 18129-18133 (2010).

31. H. S. Hillen, D. Temiakov, P. Cramer, Structural basis of mitochondrial transcription. Nat Struct Mol Biol 25, 754-765 (2018).

32. A. Rubio-Cosials et al., Human mitochondrial transcription factor A induces a U-turn structure in the light strand promoter. Nature Structural Molecular Biology 18, 1281 (2011).

33. R. Ringel et al., Structure of human mitochondrial RNA polymerase. Nature 478, 269-273 (2011).

34. F. Sciortino, R. Bansil, H. E. Stanley, P. Alstrom, Interference of phase separation and gelation: A zeroth-order kinetic model. Phys Rev E Stat Phys Plasmas Fluids Relat Interdiscip Topics 47, 4615-4618 (1993).

35. S. Boeynaems et al., Spontaneous driving forces give rise to protein-RNA condensates with coexisting phases and complex material properties. Proc Natl Acad Sci US A 116, 7889-7898 (2019).

36. A. A. Hyman, C. A. Weber, F. Jülicher, Liquid-liquid phase separation in biology. Annual review of cell and developmental biology 30, 39-58 (2014).

37. J. D. Schmit, M. Feric, M. Dundr, How Hierarchical Interactions Make Membraneless Organelles Tick Like Clockwork. Trends Biochem Sci 46, 525-534 (2021).

38. A. Sarfallah, D. Temiakov, in Mitochondrial Gene Expression. (Springer, 2021), pp. 3541.

39. I. Alshareedah, M. M. Moosa, M. Raju, D. A. Potoyan, P. R. Banerjee, Phase transition of RNA- protein complexes into ordered hollow condensates. Proceedings of the National Academy of Sciences 117, 15650-15658 (2020).

40. A. A. Jourdain, E. Boehm, K. Maundrell, J.-C. Martinou, Mitochondrial RNA granules: compartmentalizing mitochondrial gene expression. Journal of Cell Biology 212, 611-614 (2016). 
41. A. P. West et al., Mitochondrial DNA stress primes the antiviral innate immune response. Nature 520, 553-557 (2015).

42. M. Pellegrini et al., MTERF2 is a nucleoid component in mammalian mitochondria. Biochimica et Biophysica Acta (BBA)-Bioenergetics 1787, 296-302 (2009).

43. D. Spadafora, N. Kozhukhar, V. N. Chouljenko, K. G. Kousoulas, M. F. Alexeyev, Methods for Efficient Elimination of Mitochondrial DNA from Cultured Cells. PLoS One 11, e0154684 (2016).

44. J. M. Choi, F. Dar, R. V. Pappu, LASSI: A lattice model for simulating phase transitions of multivalent proteins. PLoS Comput Biol 15, e1007028 (2019).

45. H. B. Ngo, G. A. Lovely, R. Phillips, D. C. Chan, Distinct structural features of TFAM drive mitochondrial DNA packaging versus transcriptional activation. Nat Commun 5, 3077 (2014).

46. D. L. J. Lafontaine, J. A. Riback, R. Bascetin, C. P. Brangwynne, The nucleolus as a multiphase liquid condensate. Nat Rev Mol Cell Biol 22, 165-182 (2021).

47. W.-K. Cho et al., Mediator and RNA polymerase II clusters associate in transcriptiondependent condensates. Science 361, 412-415 (2018).

48. J. E. Henninger et al., RNA-Mediated Feedback Control of Transcriptional Condensates. Cell 184, 207-225.e224 (2021).

49. B. Beckert, B. Masquida, in Rna. (Springer, 2011), pp. 29-41.

50. W. Peeples, M. K. Rosen, Mechanistic dissection of increased enzymatic rate in a phaseseparated compartment. Nature chemical biology 17, 693-702 (2021).

51. L. B. Case, X. Zhang, J. A. Ditlev, M. K. Rosen, Stoichiometry controls activity of phaseseparated clusters of actin signaling proteins. Science 363, 1093-1097 (2019).

52. M. Dundr et al., A kinetic framework for a mammalian RNA polymerase in vivo. Science 298, 1623-1626 (2002).

53. L. A. Belfiore, Transport phenomena for chemical reactor design. (John Wiley \& Sons, 2003).

54. Y.-T. Tu, A. Barrientos, The human mitochondrial DEAD-box protein DDX28 resides in RNA granules and functions in mitoribosome assembly. Cell reports 10, 854-864 (2015).

55. C. Brüser, J. Keller-Findeisen, S. Jakobs, The TFAM-to-mtDNA ratio defines innercellular nucleoid populations with distinct activity levels. Cell Rep 37, 110000 (2021).

56. H. Yu et al., HSP70 chaperones RNA-free TDP-43 into anisotropic intranuclear liquid spherical shells. Science 371, (2021).

57. A. Shakya, J. T. King, DNA Local-Flexibility-Dependent Assembly of Phase-Separated Liquid Droplets. Biophys J 115, 1840-1847 (2018).

58. A.-M. Ladouceur et al., Clusters of bacterial RNA polymerase are biomolecular condensates that assemble through liquid-liquid phase separation. Proceedings of the National Academy of Sciences 117, 18540-18549 (2020).

59. C. Mata Martin, Z. Sun, Y. N. Zhou, D. J. Jin, Extrachromosomal Nucleolus-Like Compartmentalization by a Plasmid-Borne Ribosomal RNA Operon and Its Role in Nucleoid Compaction. Front Microbiol 9, 1115 (2018).

60. Y. I. Morozov et al., A model for transcription initiation in human mitochondria. Nucleic Acids Res 43, 3726-3735 (2015).

61. M. Sologub, D. Litonin, M. Anikin, A. Mustaev, D. Temiakov, TFB2 is a transient component of the catalytic site of the human mitochondrial RNA polymerase. Cell 139, 934-944 (2009). 
62. M. E. Oates et al., $\mathrm{D}^{2} \mathrm{P}^{2}$ : database of disordered protein predictions. Nucleic Acids Res 41, D508-516 (2013).

63. K. M. Ruff, F. Dar, R. V. Pappu, Ligand effects on phase separation of multivalent macromolecules. Proceedings of the National Academy of Sciences 118, e2017184118 (2021). 


\section{Acknowledgments}

We are grateful for discussions with Misteli group members, J. Jones and M. Taylor for protein expression and purification of TFAM at the NIH/NCI/CCR Protein Production Core; and T. Karpova and D. Ball for assistance with Structured Illumination Microscopy and Laser Scanning Confocal Microscopy as part of the NIH/NCI/CCR LRBGE Optical Imaging Core. FD and RVP are also grateful to K. M. Ruff for many helpful and clarifying discussions.

\section{Funding}

Research in the Misteli lab is supported by funding from the Intramural Research Program of the National Institutes of Health (NIH), National Cancer Institute (NCI), and Center for Cancer Research (CCR) (1-ZIA-BC010309); MF is supported by a Postdoctoral Research Associate Training (PRAT) fellowship from the National Institute of General Medical Sciences (NIGMS, 1Fi2GM128585-01). DT is supported by NIH R35 GM131832. Research in the Pappu lab is supported by grants from the National Institutes of Health (R01NS121114, 5R01NS056114), the Air Force Office of Scientific Research (FA9550-20-1-0241), and the St. Jude Collaborative Research Consortium on the Biology of Membraneless Organelles.

\section{Author contributions}

MF and TM designed the research. MF performed all microscopy experiments and analysis of in vitro droplets and in vivo mitochondrial nucleoids. DT and AS designed the in vitro transcription experiments and AS performed them. FD and RVP designed the computational model. FD developed and deployed the model, performed the simulations, and analyzed the simulation results. MF, FD, RVP and TM wrote the manuscript. All authors discussed results and edited the manuscript.

\section{Competing interest}

MF, FD AS, DT and TM declare no conflict of interest. RVP is a member of the Scientific Advisory Board of Dewpoint Therapeutics Inc. The work here was not supported or influenced by this affiliation.

\section{Data and materials availability}

All data and materials are available from authors upon reasonable request.

\section{Supplementary Materials}

Materials and Methods

Figures S1-S5 
bioRxiv preprint doi: https://doi.org/10.1101/2021.12.30.474545; this version posted January 1,2022. The copyright holder for this preprint (which was not certified by peer review) is the author/funder, who has granted bioRxiv a license to display the preprint in perpetuity. It is made available under aCC-BY-NC 4.0 International license.

\section{Figures}

\section{Figure 1}
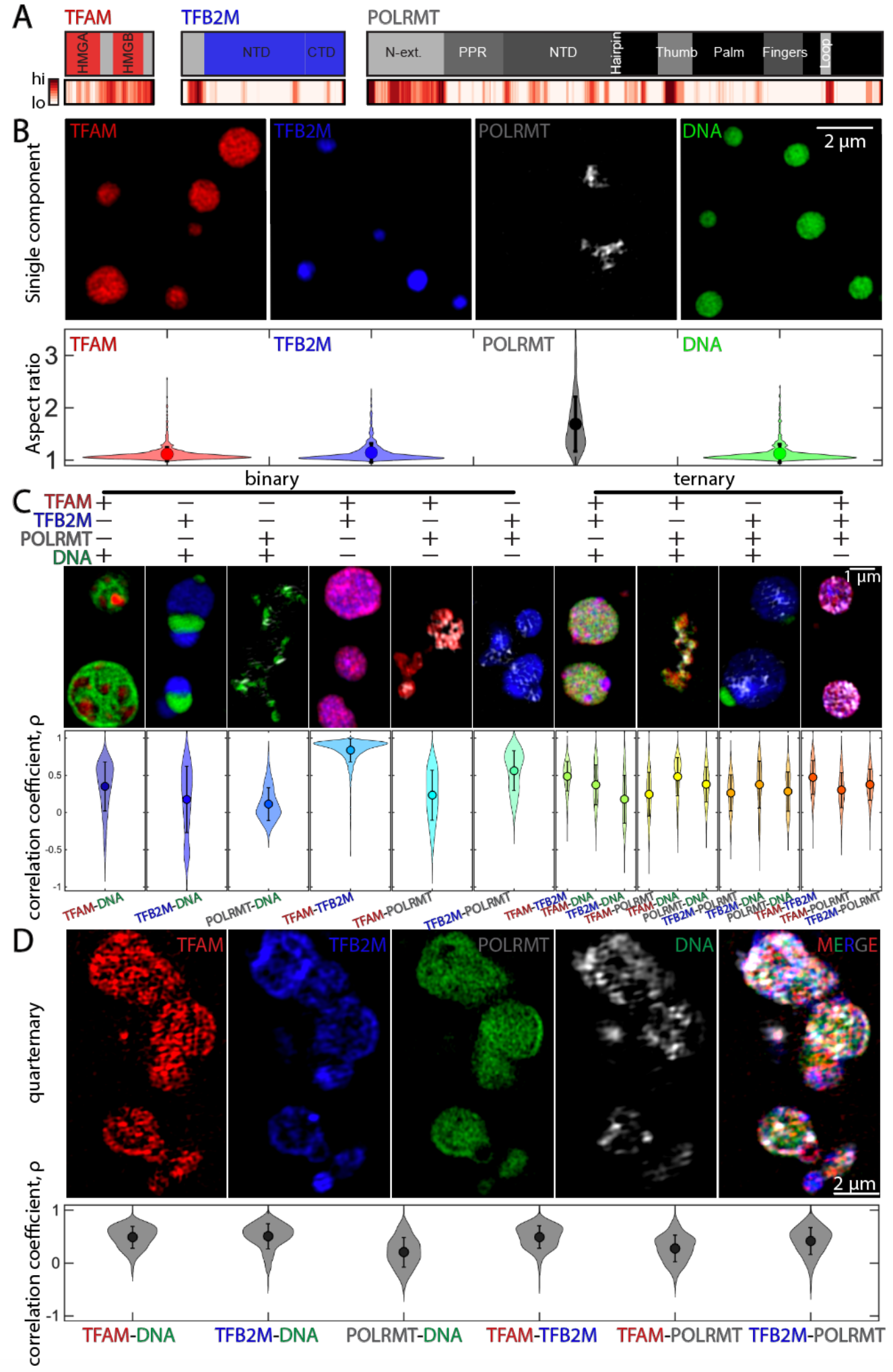
Figure 1: Equilibrium phase behavior of individual, binary, ternary, and quaternary condensates comprised of mt-transcriptional components

(A) Protein domain analysis for core mitochondrial transcription proteins TFAM, TFB2M, and POLRMT. Top row illustrates the known protein domains (red, blue, dark grey, respectively) and the unfolded, intrinsically disordered domains are indicated in light gray. TFAM contains two High Mobility Group domains (HMGA/B) separated by a disordered linker and flanked by a disordered C-tail. TFB2M contains an N-terminal domain (NTD) which adopts a methyltransferase fold, and the C-terminal domain (CTD), which consists of four alpha helices and a short, flexible "tail." POLRMT is a single-subunit DNA-dependent RNA polymerase, which is distantly related to the bacteriophage T7 RNAP. It contains a hand shaped CTD that harbors the catalytic site POLRMT also contains an N-terminal domain attached to the catalytic core via a poly-proline linker. Bottom row shows probability of intrinsically disordered sequences as predicted by several models using $\mathrm{D}^{2} \mathrm{P}^{2}$, where high and low likelihoods for disorder are indicated in red (high) and white (low). (B) SIM images of condensates formed for individual components at room temperature on pluronic-treated coverslips: $10 \mu \mathrm{M}$ TFAM in 10\% PEG (red), $10 \mu \mathrm{M}$ TFB2M in 10\% PEG (blue), $1.5 \mu \mathrm{M}$ POLRMT in 5\% PEG (gray), and $500 \mathrm{nM}$ DNA in 10\% PEG (green). Bottom row contains violin plots of the aspect ratio for all condensates analyzed. $\mathrm{n}=4$ experimental replicates, average (dot) values are indicted, error bar represents the standard deviation. Scale bar $=2 \mu \mathrm{m}$. (C) Binary and ternary compound droplets. Violin plots of correlation coefficient measured for each pair of channels; correlation coefficient $=1$ denotes complete colocalization. Scale bar $=1 \mu \mathrm{m} . \mathrm{n}=4$ experimental replicates, average (dot) values are indicted, and error bar represents the standard deviation. (See Table 1 for concentrations). (D) Quaternary droplets at room temperature. Top row includes individual channels of $\sim 6 \mu \mathrm{M}$ TFAM (red), $\sim 6$ $\mu \mathrm{M}$ TFB2M (blue), $\sim 6 \mu \mathrm{M}$ POLRMT (gray), and $\sim 500 \mathrm{nM}$ DNA (green), and the merged image in 5\% PEG. Scale bar $=2 \mathrm{um}$. Bottom row contains violin plots of the correlation coefficient for all pairs of channels. $n=3$ experimental replicates, average (dot) values are indicted, and error bar represents the standard deviation. Buffer for all conditions was $20 \mathrm{mM}$ Tris-HCl, $\mathrm{pH} \sim 8.0,20 \mathrm{mM}$ $\mathrm{BME}, 10 \mathrm{mM} \mathrm{MgCl}_{2}$, and $\sim 100 \mathrm{mM} \mathrm{NaCl}$ at room temperature. 


\section{Figure 2}
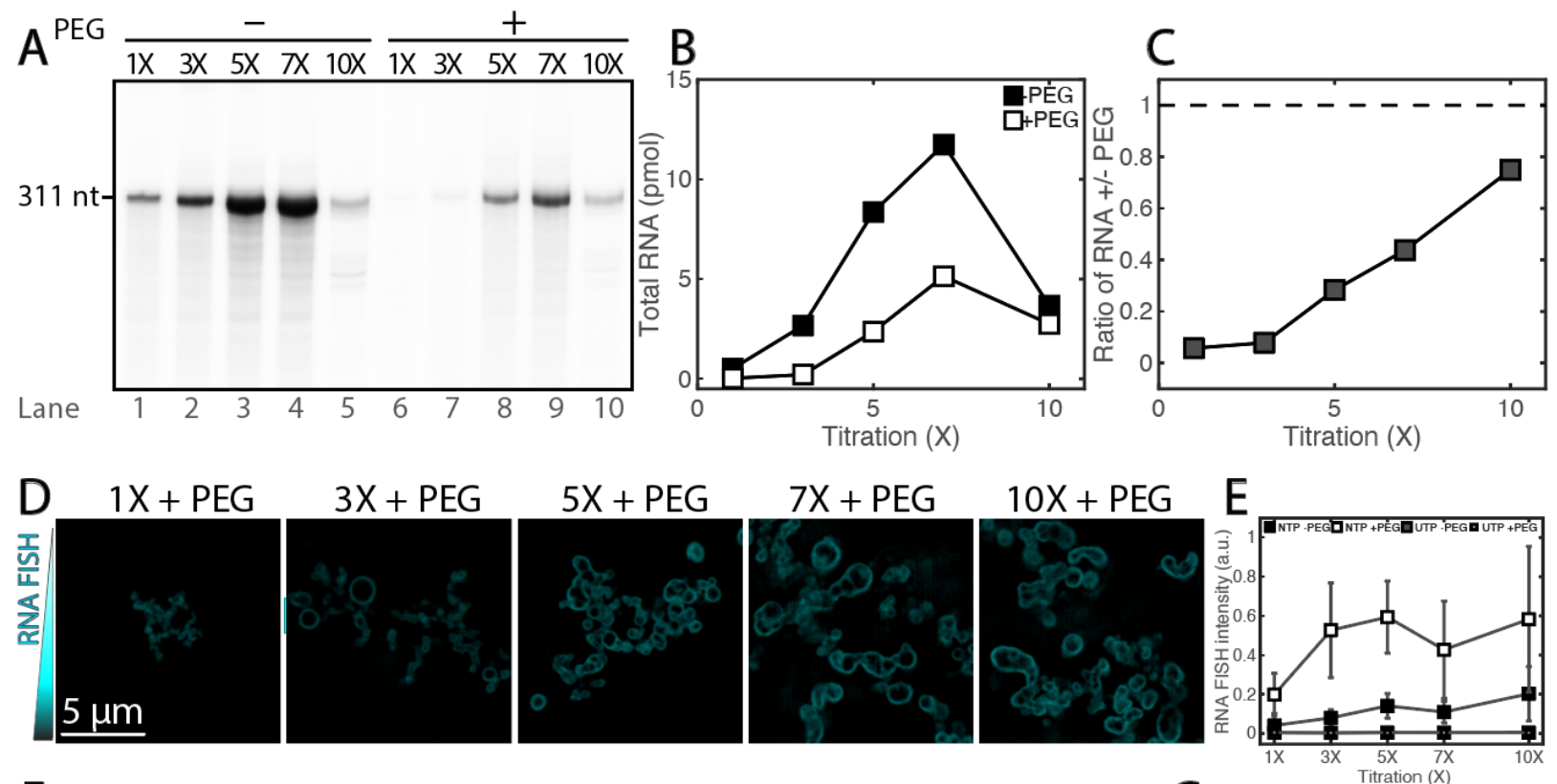

$\mathrm{F}$

DNA

RNA FISH
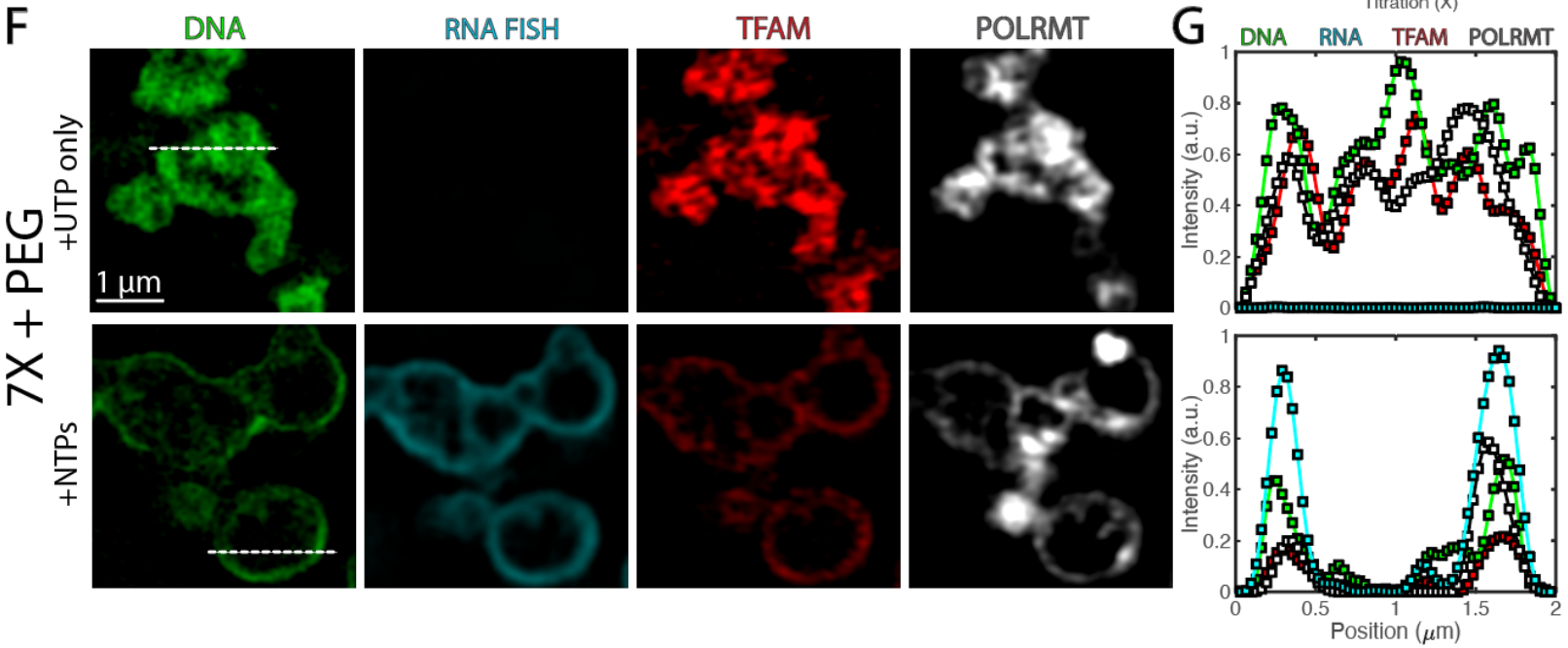

Figure 2: In vitro transcription under soluble and condensed conditions leads to changes in condensate organization

(A) RNA production rates at soluble and condensed states. The transcription run-off assay was performed using the increasing concentrations (1X to $10 \mathrm{X}$ ) of the transcription initiation complex in the absence (lanes 1-5) or presence (lanes 6-10) of PEG. Note that the apparent decrease of the transcription efficiency in lanes 5 and 10 was due, in part, to the dilution of $\left[\alpha{ }^{32} \mathrm{P}\right] \mathrm{UTP}$ with "cold" UTP. (B) Quantification of RNA product from (A) as a function of reactant titration where $0 \%$ PEG is filled squares and 5\% PEG is open squares. (C) Ratio of RNA production of crowded (5\% PEG) to soluble ( $0 \%$ PEG) as a function of reactant titration. Values in A-C illustrate a representative experiment. (D) SIM images of reactions after fixation and RNA FISH, following 1 hour of reactions under the same conditions as (A). Scale bar $=5 \mu \mathrm{m}$. All images are at the same contrast settings. (E) Quantification of RNA FISH intensity under soluble ( $\% \%$ PEG) or condensed conditions (5\% PEG) for the reactions (NTPs) and of the negative control (UTP). $\mathrm{n}=3$ experimental replicates, values represent averages and error bars represent standard deviation. (F) 
SIM images of core transcription components in condensates at $7 \mathrm{X}$ and 5\% PEG for negative control (UTP only) and for reactions (NTPs), where DNA is in green, RNA FISH is in cyan, TFAM is in red, and POLRMT is in gray scale. Scale bar $=1 \mu \mathrm{m}$. Dashed lines indicate line profile. $(\mathrm{G})$ Smoothed line profile for all components from $(\mathrm{F})$. 
bioRxiv preprint doi: https://doi.org/10.1101/2021.12.30.474545; this version posted January 1, 2022. The copyright holder for this preprint (which was not certified by peer review) is the author/funder, who has granted bioRxiv a license to display the preprint in perpetuity. It is made available under aCC-BY-NC 4.0 International license.

Figure 3

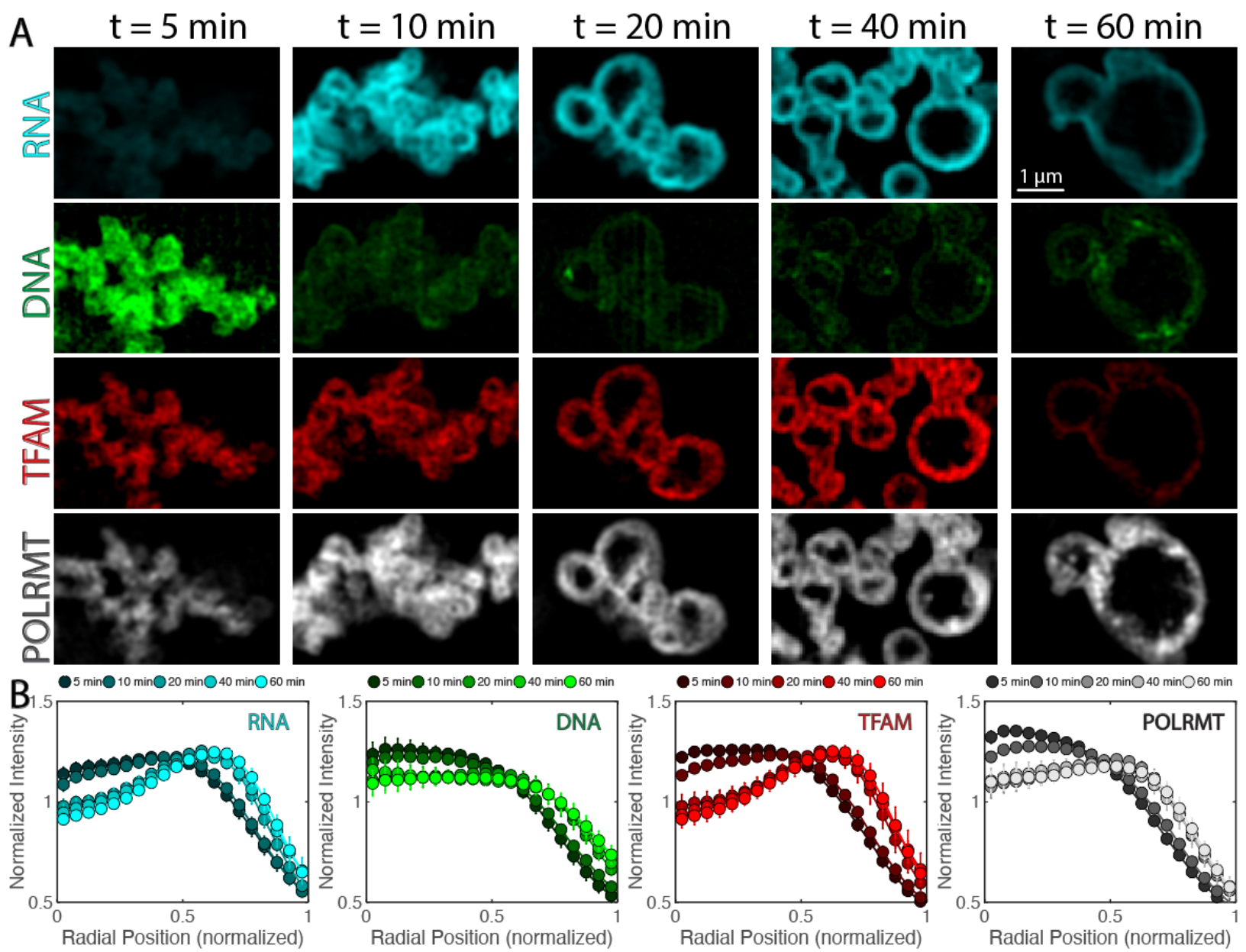

C ex-RNA at $\mathrm{t}=0 \mathrm{~min}$ ex-RNA at $\mathrm{t}=5 \mathrm{~min}$ ex $-\mathrm{RNA}$ at $\mathrm{t}=30 \mathrm{~min}$

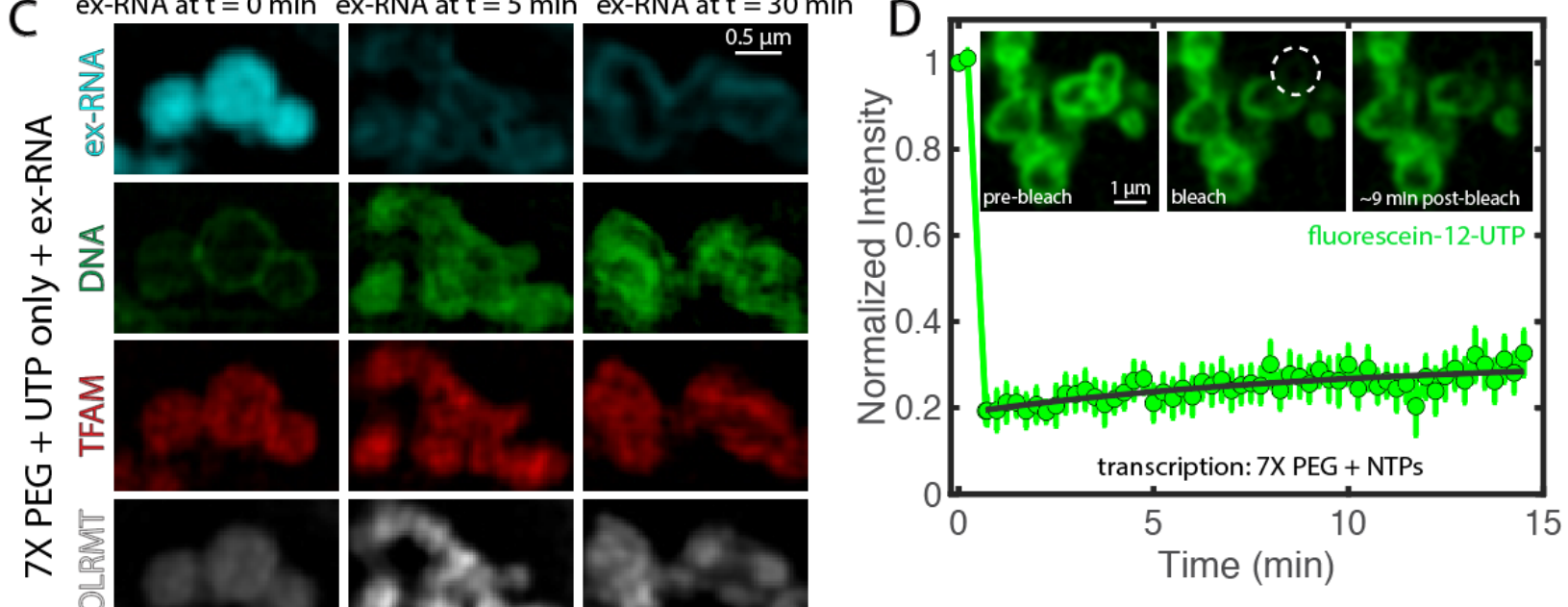




\section{Figure 3: Dynamics of transcriptional condensate organization}

(A) Time course of condensate morphology under reaction conditions (7X PEG, 5\% PEG). Condensates were fixed and image after $\mathrm{t}=5,10,20,40$, or 60 minutes at $35^{\circ} \mathrm{C}$. Single channels for RNA (cyan), DNA (green), TFAM (red), and POLRMT (grayscale) are shown. $\mathrm{n}=4$ experimental replicates and scale bar $=1 \mu \mathrm{m}$. Intensity of green channel was adjusted for visibility due to decrease in DAPI signal with time; all other channels are set at the same contrast settings. (B) Quantification of intensity profile of each component in the condensate, where $r=0$ is the center of the condensate and $r=1$ is the normalized perimeter, for each channel in (A). Shading indicates the time point corresponding to the average line profile, where darker colors are early time points and lighter colors are late time points. $n=4$ experimental replicates and error bars $=$ standard error of the mean. (C) Organization of condensates after addition of exogenous RNA (see Methods) to non-reacting droplets at $\mathrm{t}=0$ (RNA added before all other proteins/DNA), $\mathrm{t}=5$ minutes (RNA added after condensates assembled $5 \mathrm{~min}$ at $35^{\circ} \mathrm{C}$ ) or $\mathrm{t}=30 \mathrm{~min}$ (RNA added after condensates assembled $30 \mathrm{~min}$ at $35^{\circ} \mathrm{C}$ ). Buffer was the same as that used in the negative control ( $8 \mathrm{mM} \mathrm{UTP}$ ). Condensates were fixed onto coverslips after 1 hour of incubation at $35^{\circ} \mathrm{C} . \mathrm{n}=3$ experimental replicates and scale bar $=0.5 \mu \mathrm{m}$. (D) FRAP recovery for transcribing droplets (NTPs, each $2 \mathrm{mM}$ ) for $7 \mathrm{X}$ and 5\% PEG conditions. Inset shows condensates pre-bleach, bleach, and $9 \mathrm{~min}$ post-bleach. Dashed circle represents region that was bleached. Scale bar $=1 \mu \mathrm{m} . \mathrm{n}=9$ droplets and error bars $=$ standard error of the mean. 


\section{Figure 4}

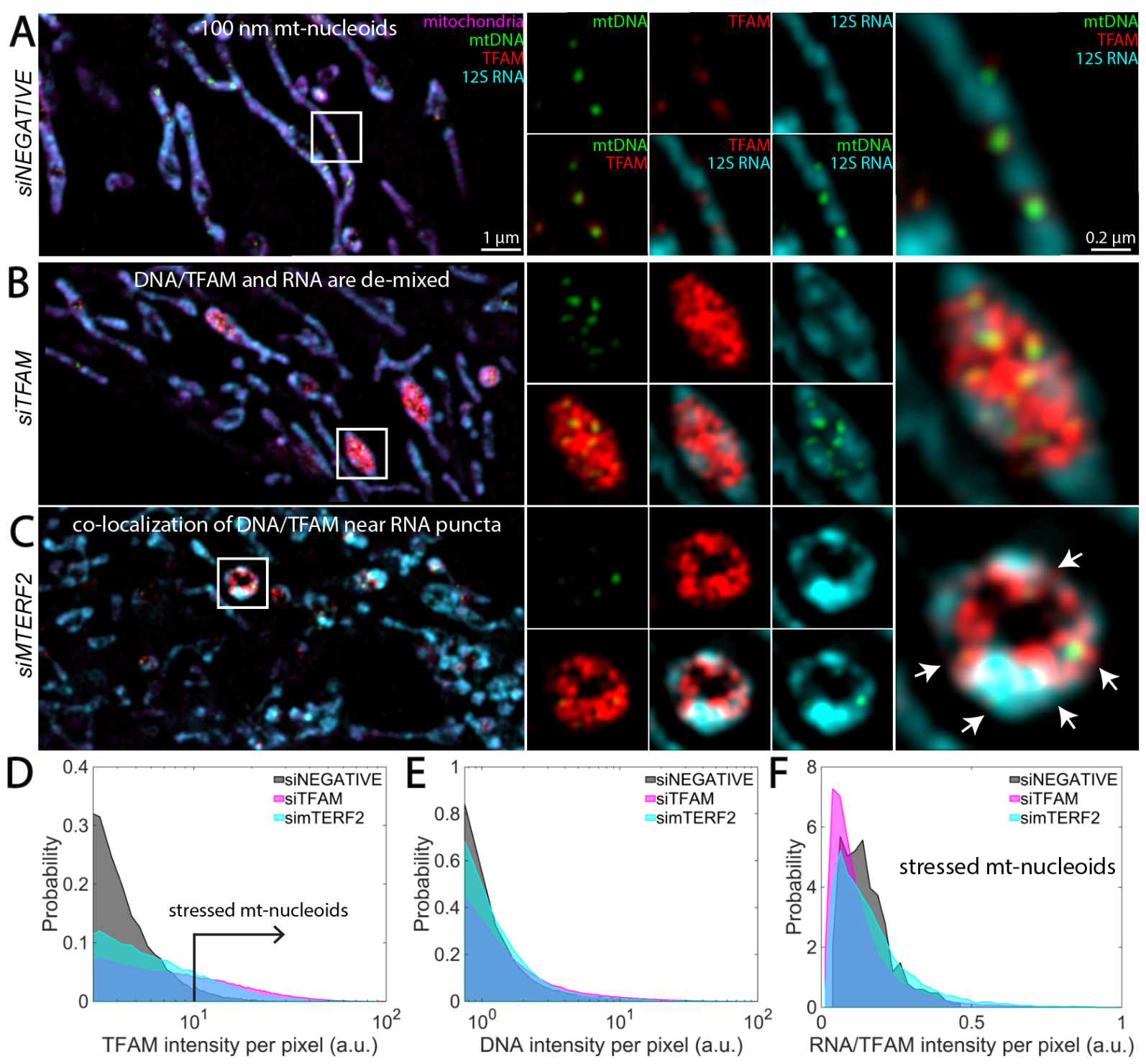

Figure 4: Structural organization upon perturbation of mt-nucleoid components in live cells

(A-C) SIM images of mitochondrial components after 72 hours of siRNA treatment: siNEGATIVE (A), siTFAM (B), and simTERF2 (C). Leftmost panels are the merged image of the zoomed-out version of the mitochondrial network, where mitochondria are in magenta (MitoTracker Red), mtDNA is in green (anti-DNA), TFAM is in red (anti-TFAM), and 12S RNA is in cyan (RNA FISH). Scale bar $=1 \mu \mathrm{m}$. White box indicates region of interest (ROI). Middle panels are signal or two-channel overlays of the ROI. Rightmost panels are the three-channel overlay for the ROI. Scale bar $=0.2 \mu \mathrm{m}$. Arrows in (C) indicate mtDNA puncta co-localizing with TFAM, and 12S RNA. Intensities across each channel are matched across siRNA conditions. $n=4$ independent experimental replicates. (D) Probability distribution of TFAM pixel intensity within a segmented nucleoid, where gray $=$ siNEGATIVE, magenta $=$ siTFAM, and cyan $=$ siMTERF2. Nucleoids 
containing higher than normal TFAM intensities are considered to be stressed as indicated by black arrow. (E) Probability distribution of DNA pixel intensity within a segmented nucleoid, where gray $=$ siNEGATIVE, magenta $=$ siTFAM, and cyan = siMTERF2. (F) RNA/TFAM intensity for stressed nucleoids (see arrow in D). Data represent $n=4$ independent experimental replicates that were pooled together. 
bioRxiv preprint doi: https://doi.org/10.1101/2021.12.30.474545; this version posted January 1 2022. The copyright holder for this preprint (which was not certified by peer review) is the author/funder, who has granted bioRxiv a license to display the preprint in perpetuity. It is made available under aCC-BY-NC 4.0 International license.

Figure 5:

\section{A}

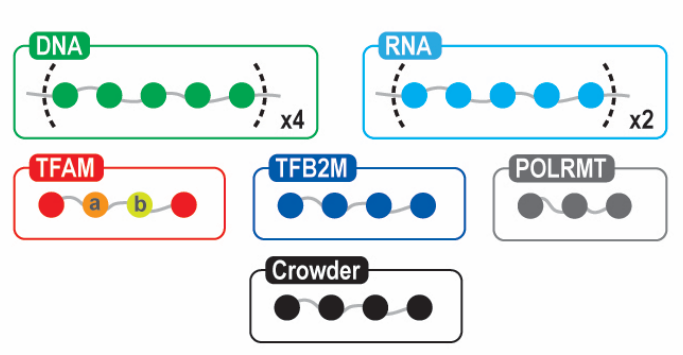

B

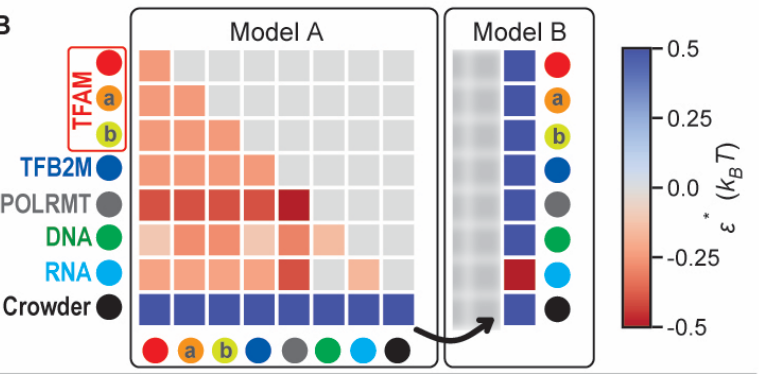

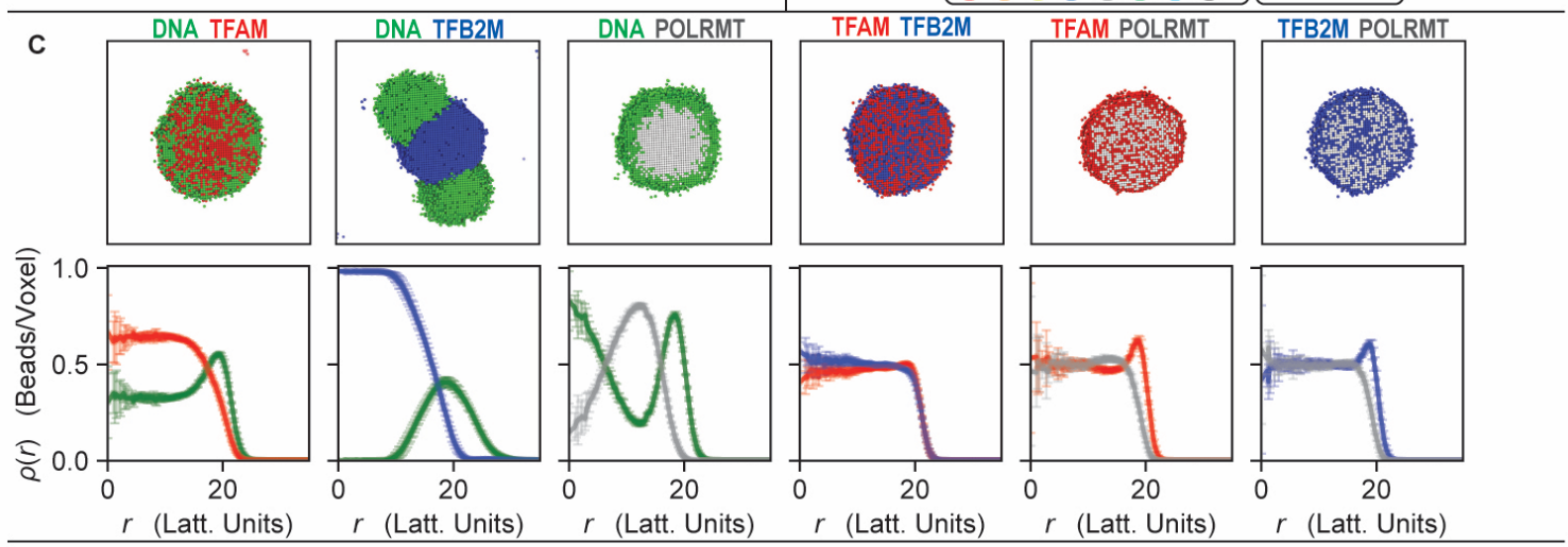

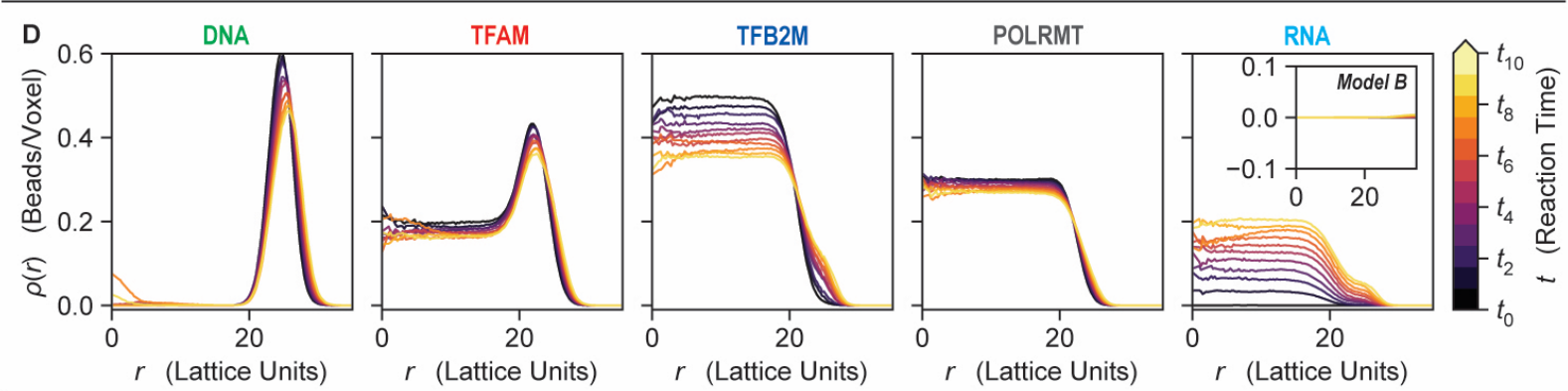

E

Model A: No RNA Binding Proteins
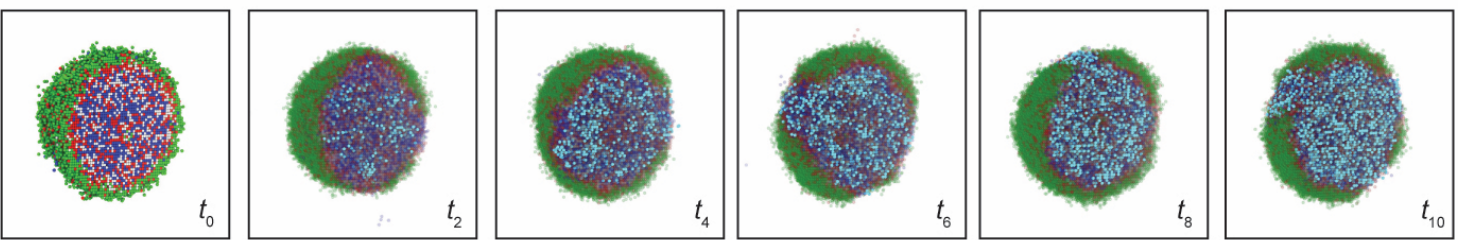

Reaction Time

$\mathbf{F}$
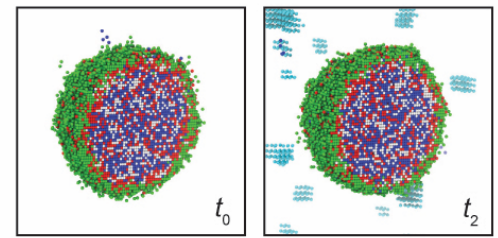

Model B: With RNA Binding Proteins
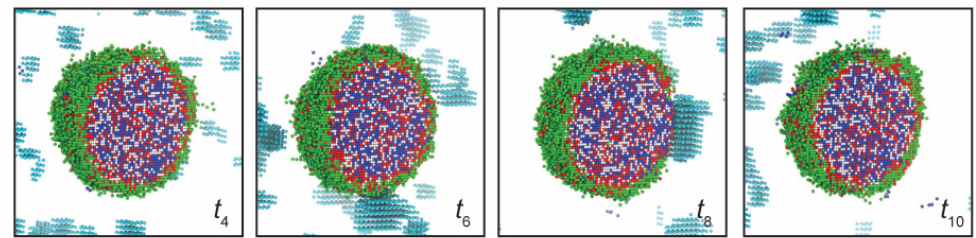

Reaction Time 


\section{Figure 5: Computational modelling of mitochondrial transcriptional condensates}

(A) Coarse-grained model for the mitochondrial transcriptional components. All beads are connected by implicit linkers of 2-lattice sites. DNA is modeled as chains of 20 beads. Since the mtDNA template used in the experiments produces RNA about half as long as the DNA, the RNA is a chain of 10 beads. TFAM is modeled as 4 beads (' $X$ - $a-b-X$ ') where the central two beads, denoted as beads $a$ and $b$, interact more favorably with DNA. To account for TFAM's weak dimerization upon DNA binding, bead $b$ has an additional interaction energy $\left(-2 \mathrm{k}_{\mathrm{B}} \mathrm{T}\right)$ for another $b$ bead, yielding a local anisotropic interaction. TFB2M is represented as 4 beads, and POLRMT is represented as 3 beads due to the highly folded nature of the molecule. Lastly, the Crowder is represented as a chain of 4 beads. (B) Interaction matrices for the two models considered. Model A lacks RNA-binding proteins, and recaptures the organization of the condensates seen in vitro. The Crowder has repulsive interactions with every species, including itself. The DNA and RNA have no interactions, while the rest of the molecules have favorable interactions. Model B mimics the effective inclusion of RNA-binding proteins by making the RNA-Crowder interactions to be favorable, modeling an effective RNA binding protein. (C) Representative snapshots and density profiles for the binary mixtures shown in Fig. 1C. For clarity, the Crowder is not shown. Model A qualitatively captures the experimental images. DNA \& TFAM form mixed condensates with TFAM having smaller droplets within. DNA \& TFB2M generate droplets that do not fully colocalize but stay closely associated. TFAM \& TFB2M form well mixed droplets. POLRMT mixtures have dense POLRMT regions covered by the other component. (D) Density profiles of each component with different RNA amounts. DNA is pushed outwards as RNA is increased. For TFAM the interior most density does not change but the density at the outer rim shared with DNA is reduced. TFB2M is significantly pushed outwards as RNA is increased. RNA is accumulated evenly inside the interior of the droplet as RNA is increased. The inset corresponds to Model B and shows that no RNA is accumulated inside the condensate when simulated RNA binding proteins are present. (E) Representative snapshots of the condensates with increasing RNA for Model A. A higher amount of RNA in the system corresponds to a later time in the transcriptional reactions. For clarity, the Crowder is not shown, and non-RNA components are made transparent. The RNA evenly distributes itself inside the condensates and continues to be accumulated as the reaction continues. (F) Representative snapshots like (E) but for Model B. With a suitable RNA binding protein, the RNA can be prevented from going inside the condensates. 